\title{
Ultrahigh-Resolution Magnetic Resonance in Inhomogeneous Magnetic Fields: Two-Dimensional Long-Lived-Coherence Correlation Spectroscopy
}

\author{
Srinivas Chinthalapalli, ${ }^{1}$ Aurélien Bornet, ${ }^{2}$ Takuya F. Segawa, ${ }^{2}$ Riddhiman Sarkar, ${ }^{3}$ \\ Sami Jannin, ${ }^{2, *}$ and Geoffrey Bodenhausen ${ }^{2,4,5,6}$ \\ ${ }^{1}$ Department of Chemistry, Indian Institute of Technology, 110016 New Delhi, India \\ ${ }^{2}$ Institut des Sciences et Ingénierie Chimiques, Ecole Polytechnique Fédérale de Lausanne, 1015 Lausanne, Switzerland \\ ${ }^{3}$ Departement Chemie, Technische Universität München, Lichtenbergstraße 4, D-85747 Garching, Germany \\ ${ }^{4}$ Département de Chimie, Ecole Normale Supérieure, 24 rue Lhomond, 75231 Paris cedex 05, France \\ ${ }^{5}$ Université Pierre et Marie Curie, 75005 Paris, France \\ ${ }^{6}$ UMR 7203, CNRS/UPMC/ENS, 75005 Paris, France
}

(Received 2 April 2012; published 26 July 2012)

\begin{abstract}
A half-century quest for improving resolution in Nuclear Magnetic Resonance (NMR) and Magnetic Resonance Imaging (MRI) has enabled the study of molecular structures, biological interactions, and fine details of anatomy. This progress largely relied on the advent of sophisticated superconducting magnets that can provide stable and homogeneous fields with temporal and spatial variations below $\Delta \mathrm{B}_{0} / \mathrm{B}_{0}<0.01 \mathrm{ppm}$. In many cases however, inherent properties of the objects under investigation, pulsating arteries, breathing lungs, tissue-air interfaces, surgical implants, etc., lead to fluctuations and losses of local homogeneity. A new method dubbed "long-lived-coherence correlation spectroscopy" (LLC-COSY) opens the way to overcome both inhomogeneous and homogeneous broadening, which arise from local variations in static fields and fluctuating dipole-dipole interactions, respectively. LLC-COSY makes it possible to obtain ultrahigh resolution two-dimensional spectra, with linewidths on the order of $\Delta \nu=0.1$ to $1 \mathrm{~Hz}$, even in very inhomogeneous fields $\left(\Delta \mathrm{B}_{0} / \mathrm{B}_{0}>10 \mathrm{ppm}\right.$ or $5000 \mathrm{~Hz}$ at $\left.9.7 \mathrm{~T}\right)$, and can improve resolution by a factor up to 9 when the homogeneous linewidths are determined by dipole-dipole interactions. The resulting LLC-COSY spectra display chemical shift differences and scalar couplings in two orthogonal dimensions, like in " $J$ spectroscopy." LLC-COSY does not require any sophisticated gradient switching or frequency-modulated pulses. Applications to in-cell NMR and to magnetic resonance spectroscopy (MRS) of selected volume elements in MRI appear promising, particularly when susceptibility variations tend to preclude high resolution.
\end{abstract}

DOI: 10.1103/PhysRevLett.109.047602

PACS numbers: 82.56.Fk, 82.56.Pp, 82.56.Jn

Nuclear magnetic resonance (NMR) is often regarded as one of the most versatile forms of spectroscopy. Magnetic resonance imaging (MRI) has offered new insights into morphology, metabolism, blood flow, diffusion, and brain activity. Most applications to inanimate samples and living organisms rely on the use of very homogeneous magnetic fields $B_{0}$ with spatial variations below $1 \mathrm{ppb}\left(10^{-9}\right)$, so that subtle differences in the environments of various nuclei, chemical shifts and couplings can be observed. This makes NMR costly and cumbersome. In many cases, inanimate samples or living organisms under investigation are inherently heterogeneous and suffer from variations of susceptibility due to voids or surgical implants [1,2]. Animals and human beings cannot be kept still because of pulsating arteries and respiration. Very high fields beyond $23 \mathrm{~T}$ can only be generated by nonpersistent resistive or hybrid magnets $[3,4]$ which are driven by unstable power supplies and suffer from vibrations due to their cooling systems. As a result, the magnetic fields may suffer from temporal and spatial fluctuations. In some cases, bulky samples ranging from frescoes to glaciers, from shales to living trees cannot be inserted in a magnet bore [5,6]. We show in this contribution how ultra-high-resolution NMR spectra can be obtained in inhomogeneous fields with unknown spatiotemporal variations. Many techniques have been proposed to acquire high-resolution spectra under adverse conditions. Spin echoes [7,8] can refocus the effects of inhomogeneous $\mathrm{B}_{0}$ fields, and echo modulations can reveal hidden spin-spin couplings [9-11]. If the field's spatial distribution is known, the $\mathrm{B}_{0}$ inhomogeneity may be compensated by using a radio-frequency (rf) field with a spatial profile $\mathrm{B}_{1}(\mathbf{r})$ designed to match the profile $\mathrm{B}_{0}(\mathbf{r})$ of the static field [12-14], or by manipulating the phases of the magnetization vectors associated with different voxels in the presence of known gradients $[15,16]$. In either case, the field $\mathrm{B}_{0}(\mathbf{r})$ must be time independent, and its spatial profile must be known. We have recently $[17,18]$ obtained highresolution spectra and images in inhomogeneous fields by tracking the differences of the precession frequencies of two spins $[19,20]$, in the manner of "ultrafast" methods developed by the teams of Frydman [21], Pelupessy [22] and Giraudeau [23]. Here we describe a novel NMR method that yields ultra-high-resolution two-dimensional (2D) spectra, even in very inhomogeneous and spatiotemporally 
variable magnetic fields. The new NMR method cancels the effects of inhomogeneous broadening, is insensitive to local magnetic field fluctuations, and can reduce homogeneous broadening caused by homo-nuclear dipole-dipole couplings by a factor up to 9 . In a system with two scalarcoupled spins $I=1 / 2$ and $S=1 / 2$ (e.g., two nonequivalent protons), one can excite long-lived coherences (LLC's) that can have very long lifetimes $T_{\text {LLC }}$ (much longer than the transverse relaxation times $T_{2}$ ) and hence yield very narrow linewidths $\left.\Delta \nu_{\mathrm{LLC}}=1 /\left(\pi T_{\mathrm{LLC}}\right)\right)$ [24,25]. Their precession frequencies are independent of offset, and hence of chemical shifts and inhomogeneous broadening, and are determined by the total coupling $T_{I S}$, i.e., by the sum of scalar and residual dipolar couplings $\left(T_{I S}=2 J_{I S}+\right.$ $\left.D_{I S}\right)$. In this work, LLC's are excited and detected over a wide range of frequencies of about $5 \mathrm{kHz}$, thus covering the entire ${ }^{1} \mathrm{H}$ spectrum despite the inhomogeneous field. The 2D spectra display chemical shift differences in a first $\omega_{1}$ dimension and total couplings $T_{I S}=2 J_{I S}+D_{I S}$ in a second $\omega_{2}$ dimension.

Long-lived coherences (LLC's) are closely related to long-lived states (LLS) [26-28]. In a simple system consisting of a pair of inequivalent coupled spins $I=1 / 2$ and $S=1 / 2$, singlet and triplet states arise from linear combinations of the four product states: $S_{0}=1 / \sqrt{2}(|\alpha \beta\rangle-$ $|\beta \alpha\rangle), \quad T_{+1}=|\alpha \alpha\rangle, \quad T_{0}=1 / \sqrt{2}(|\alpha \beta\rangle+|\beta \alpha\rangle) \quad$ and $T_{-1}=|\beta \beta\rangle$. The LLS is defined as the difference between the population of the singlet state and the average population of the three triplet states, and has a very long lifetime [29] because of its immunity to fluctuations of intramolecular dipolar interactions $D_{I S}$. LLS are ideal to "store" magnetization over remarkably long time scales and, among other things, allow one to determine very slow diffusion coefficients. [30]

A long-lived coherence (LLC) is a coherent superposition between a long-lived singlet state $S_{0}$ and a triplet state $T_{0}$ and therefore belongs to the class of zero-quantum coherences. When expressed in the singlet-triplet base, an LLC is defined as a linear combination of $Q_{\mathrm{LLC}}=$ $\left|S_{0}\right\rangle\left\langle T_{0}|+| T_{0}\right\rangle\left\langle S_{0}\right|$ and $\left|S_{0}\right\rangle\left\langle T_{0}|-| T_{0}\right\rangle\left\langle S_{0}\right|$, which are equivalent to $Q_{\mathrm{LLC}}=I_{x}-S_{x}$ and $2 I_{y} S_{z}-2 I_{z} S_{y}$ in the product base [25]. Such coherences can easily be excited and sustained using conventional NMR spectrometers. LLC's slowly decay with long time constants $T_{\text {LLC }}=$ $1 / R_{\mathrm{LLC}}=\kappa T_{2}$ where $3 \leq \kappa \leq 9[25,31]$ and are modulated by the total coupling $T_{I S}=2 J_{I S}+D_{I S}$ :

$$
\begin{aligned}
Q_{\mathrm{LLC}}(t)= & {\left[\left(I_{x}-S_{x}\right) \cos \left(\pi T_{I S} t\right)\right.} \\
& \left.+\left(2 I_{y} S_{z}-2 I_{z} S_{y}\right) \sin \left(\pi T_{I S} t\right)\right] \exp \left(-R_{\mathrm{LLC}} t\right) .
\end{aligned}
$$

This slow oscillatory decay, when subjected to a Fourier transform, leads to dramatically enhanced resolution compared to conventional NMR with linewidths $1 /\left(\pi T_{\mathrm{LLC}}\right)=1 /\left(\pi \kappa T_{2}\right) \ll 1 /\left(\pi T_{2}\right)$. Since LLC's belong to the class of zero-quantum coherences, they are immune to inhomogeneous broadening. We recently designed a fast "on-the-fly" acquisition scheme for LLC's [32] by briefly interrupting the rf irradiation required to sustain the LLC's at regular intervals, so that they are ephemerally transformed into observable single-quantum coherences (SQC's). This scheme offers substantial gains in acquisition times, albeit at the price of a loss of signal-to-noise ratio (SNR) which decreases as the square root of the duty cycle of signal acquisition.

Both the original high-field experiment [24] and the onthe-fly method [32] started by exciting LLC's via $I_{x}-S_{x}$. Such excitation schemes strongly depend on the carrier frequency and cannot be used in inhomogeneous fields. We therefore developed a new excitation scheme via zeroquantum coherences. In the sequence of Fig. 1, a zeroquantum coherence $Z Q_{x}$ is prepared by three pulses with intervals $\tau=1 /\left(4 J_{I S}\right)$. The resulting $Z_{x}$ evolves during the evolution interval $t_{1}$ so that after applying a $(\pi / 2)_{y}$ pulse at point (5), only magnetization stemming from the term $\mathrm{ZQ}_{y}=\frac{1}{2}\left(2 I_{y} S_{x}-2 I_{x} S_{y}\right)$ is transformed into LLC's that can be sustained by rf irradiation:

$$
\sigma_{(5)}=\left(2 I_{y} S_{z}-2 I_{z} S_{y}\right) \sin \left(2 \pi \Delta \nu_{I S} t_{1}\right) .
$$

In this excitation scheme, the LLC amplitude is modulated by the chemical shift difference $\Delta \nu_{I S}$ in the $t_{1}$ evolution interval.

It is then straightforward to carry out a two-dimensional experiment by incrementing $t_{1}$ to monitor the modulation of the LLC's which is due to the factor $\sin \left(2 \pi \Delta \nu_{I S} t_{1}\right)$, thus providing information about chemical shift differences $\Delta \nu_{I S}$. The 2D spectrum is insensitive to inhomogeneous broadening in both $\omega_{1}$ and $\omega_{2}$ dimensions.

The remarkable properties of the novel 2D LLC-COSY experiment were illustrated by spectra of a mixture of $0.1 \mathrm{M}$ alanine-glycine (AlaGly or molecule 1 ) and $0.1 \mathrm{M}$ 3-bromothiophene-2-carboxylic acid (BTCA or molecule 2) in $\mathrm{D}_{2} \mathrm{O}$ in a magnetic field of $11.7 \mathrm{~T}$, corresponding to a ${ }^{1} \mathrm{H}$ frequency of $500 \mathrm{MHz}$. Each of these two molecules

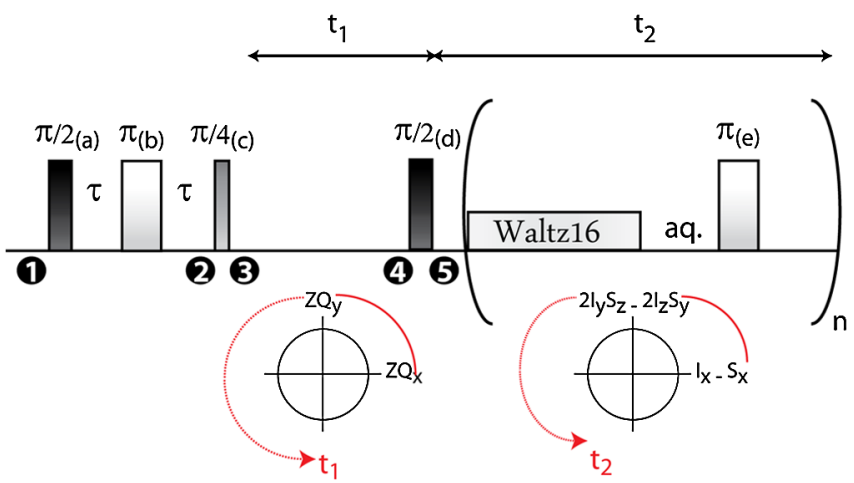

FIG. 1 (color online). Excitation and acquisition of LLC's via $\mathrm{ZQ}_{y}$. Receiver dead-times between pulses and signal acquisition intervals ( $3 \mu \mathrm{s}$ in our case) are not shown. 
a
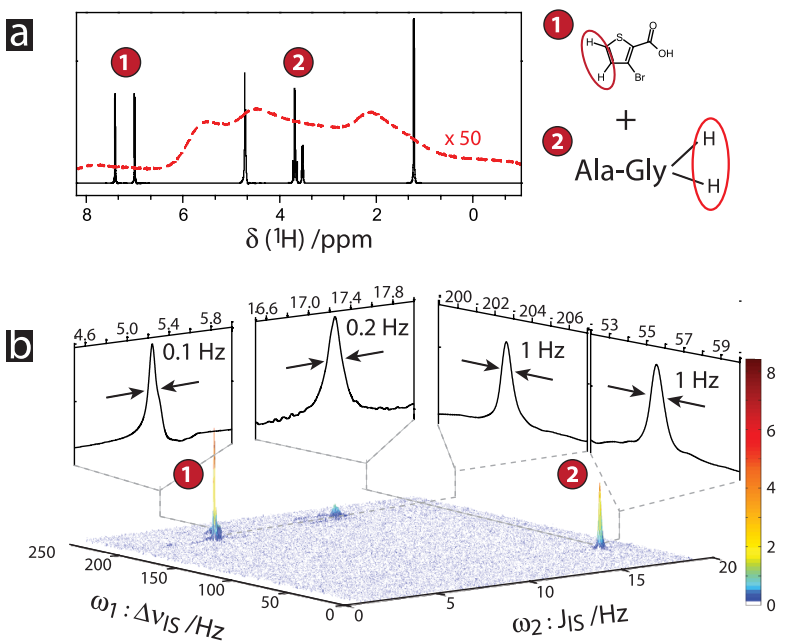

FIG. 2 (color online). (a) One-dimensional proton spectrum of a mixture of 3-bromothiophene carboxylic acid (BTCA, 1) and Alanine-Glycine (Ala-Gly, 2) at $11.7 \mathrm{~T}\left(500 \mathrm{MHz}\right.$ for $\left.{ }^{1} \mathrm{H}\right)$ in a normal homogeneous field (black solid lines) and in a very inhomogeneous field where the linewidth at half height was deliberately degraded to $\Delta \nu=3000 \mathrm{~Hz}$ (red dotted lines). (b) 2D LLC-COSY spectrum acquired in the same inhomogeneous field. The enlargements stem from cross sections extracted at the $J_{I S}$ and $\Delta \nu_{I S}$ values of molecules (1) and (2). A total of 2048 and 650 points were recorded in the direct $t_{2}$ and indirect $t_{1}$ dimensions with increments $\Delta t_{2}=50 \mathrm{~ms}$ and $\Delta t_{1}=2 \mathrm{~ms}$, respectively.

contains a pair of spins, but with distinct chemical shift differences and $J$-coupling constants: $\Delta \nu_{I S}=55.46 \mathrm{~Hz}$ and $J_{I S}=17.22 \mathrm{~Hz}$ for the two $\mathrm{H}^{\alpha}$ protons in glycine in AlaGly, and $\Delta \nu_{I S}=202.4 \mathrm{~Hz}$ and $J_{I S}=5.32 \mathrm{~Hz}$ for the two aromatic protons in BTCA.

The homogeneity of the magnetic field was deliberately degraded by setting the shim coil currents far from their optimum. The proton NMR linewidths at half height were thus artificially broadened to $\Delta \nu=3000 \mathrm{~Hz}$ [Fig. 2(a)]. The 2D LLC COSY spectrum was obtained with $\tau=$ $1 /\left(4 J_{I S}{ }^{(\mathrm{AlaGly})}\right) \approx 3 /\left(4 J_{I S}{ }^{(\mathrm{BTCA})}\right)$ to maximize the signals of both AlaGly and BTCA.

The 2D spectra in Figs. 2(b) and 2(c) show that, despite the inhomogeneity of the field, it is possible to recover chemical shift differences and $J$-coupling constants with linewidths below $1 \mathrm{~Hz}$. The peak at $\Delta \nu_{I S}=202.4 \mathrm{~Hz}$ and $J_{I S}=5.32 \mathrm{~Hz}$ corresponds to BTCA which has a very long $T_{\mathrm{LLC}}$, and hence a narrow linewidth of $1 \mathrm{~Hz}(=2 \mathrm{ppb})$ in the $\omega_{1}\left(\Delta \nu_{I S}\right)$ dimension and $0.1 \mathrm{~Hz}(=0.2 \mathrm{ppb})$ in the $\omega_{2}$ $\left(J_{I S}\right)$ dimension. The latter can be further reduced by decreasing the duty cycle of the signal acquisition, and by extending $t_{2}{ }^{\max }$. As ZQC and LLC relaxation rates arise mainly from paramagnetic interactions (hetero- or homonuclear intramolecular interactions playing only a marginal role), further line narrowing in the two dimensions can be achieved by degassing the solution or scavenging dissolved $\mathrm{O}_{2}$ with ascorbic acid [33]. An improvement in linewidth from $3 \mathrm{kHz}$ to $0.1 \mathrm{~Hz}$ results in a net increase in peak heights by a factor $3 \times 10^{4}$. AlaGly gives two peaks: one at $\left(J_{I S}=17.22 \mathrm{~Hz} ; \Delta \nu_{I S}=55.46 \mathrm{~Hz}\right)$ stemming from the two $\mathrm{H}^{\alpha}$ protons of glycine and one (folded) peak at $\left(J_{I S}=7.4 \mathrm{~Hz} ; \Delta \nu_{I S}=204 \mathrm{~Hz}\right)$ that stems from an LLC involving the $\mathrm{H}^{\alpha}$ proton of alanine and one of its three $\mathrm{H}^{\beta}$ protons. Further discussions of the broadband features of the LLC-COSY method, including experimental details, are presented in the supplemental material [34]. LLC-COSY experiments have been successfully performed in molecules of biological interest such as malic acid and in the three-spin system of acrylic acid, in very inhomogeneous magnetic fields. We believe that LLCCOSY should become a promising technique for ex-situ or in-cell NMR studies.

This novel form of 2D correlation spectroscopy exploits the unique properties of long-lived coherences (LLC's) and can afford exceptional linewidths $\left(\Delta \nu_{\mathrm{LLC}} / \nu_{0}=\right.$ $\left.2 \times 10^{-10}=0.2 \mathrm{ppb}\right)$ even in very inhomogeneous magnetic fields $\left(\Delta \mathrm{B}_{0} / \mathrm{B}_{0}=10^{-5}=10 \mathrm{ppm}\right)$.

We are indebted to Dr. Diego Carnevale and Professor Narayanan Kurur for stimulating discussions and Martial Rey for valuable technical assistance. This work was supported by the Swiss National Science Foundation (SNSF), the Swiss Commission for Technology and Innovation (CTI), and the French CNRS.

*sami.jannin@epfl.ch

[1] I. J. Cox, G. M. Bydder, D. G. Gadian, I. R. Young, E. Proctor, S. R. Williams, and I. Hart, J. Magn. Reson. 70, 163 (1986).

[2] C.E. Mountford, S. Doran, C.L. Lean, and P. Russell, Chem. Rev. 104, 3677 (2004).

[3] Y. Y. Lin, S. Ahn, N. Murali, W. Brey, C. R. Bowers, and W. S. Warren, Phys. Rev. Lett. 85, 3732 (2000).

[4] B. Shapira, K. Shetty, W. W. Brey, Z. H. Gan, and L. Frydman, Chem. Phys. Lett. 442, 478 (2007).

[5] B. Blümich, J. Perlo, and F. Casanova, Prog. Nucl. Magn. Reson. Spectrosc. 52, 197 (2008).

[6] G. Eidmann, R. Savelsberg, P. Blümler, and B. Blümich, J. Magn. Reson., Ser. A 122, 104 (1996).

[7] E. L. Hahn, Phys. Rev. 80, 580 (1950).

[8] H. Y. Carr and E. M. Purcell, Phys. Rev. 94, 630 (1954).

[9] E. L. Hahn and D.E. Maxwell, Phys. Rev. 88, 1070 (1952).

[10] R. L. Vold and S. O. Chan, J. Chem. Phys. 53, 449 (1970).

[11] R. Freeman and H. D. W. Hill, J. Chem. Phys. 54, 301 (1971).

[12] C. A. Meriles, D. Sakellariou, H. Heise, A. J. Moule, and A. Pines, Science 293, 82 (2001).

[13] J. Perlo, V. Demas, F. Casanova, C. A. Meriles, J. Reimer, A. Pines, and B. Blümich, Science 308, 1279 (2005). 
[14] V. Demas, C. Meriles, D. Sakellariou, S. I. Han, J. Reimer, and A. Pines, Concepts Magn. Reson. 29B, 137 (2006).

[15] B. Shapira and L. Frydman, J. Am. Chem. Soc. 126, 7184 (2004).

[16] B. Shapira and L. Frydman, J. Magn. Reson. 182, 12 (2006).

[17] P. Pelupessy, E. Rennella, and G. Bodenhausen, Science 324, 1693 (2009).

[18] R. Paquin, P. Pelupessy, and G. Bodenhausen, J. Magn. Reson. 201, 199 (2009).

[19] K. Nagayama, K. Wüthrich, and R. R. Ernst, Biochem. Biophys. Res. Commun. 90, 305 (1979).

[20] A. Wokaun and R. R. Ernst, Chem. Phys. Lett. 52, 407 (1977).

[21] L. Frydman, T. Scherf, and A. Lupulescu, Proc. Natl. Acad. Sci. U.S.A. 99, 15858 (2002).

[22] P. Pelupessy, J. Am. Chem. Soc. 125, 12345 (2003).

[23] P. Giraudeau and S. Akoka, J. Magn. Reson. 186, 352 (2007).

[24] R. Sarkar, P. Ahuja, P. R. Vasos, and G. Bodenhausen, Phys. Rev. Lett. 104, 053001 (2010).

[25] R. Sarkar, P. Ahuja, P. R. Vasos, A. Bornet, O. Wagnieres, and G. Bodenhausen, Prog. Nucl. Magn. Reson. Spectrosc. 59, 83 (2011).
[26] M. Carravetta and M.H. Levitt, J. Am. Chem. Soc. 126, 6228 (2004).

[27] G. Pileio and M. H. Levitt, J. Chem. Phys. 130, 214501 (2009).

[28] M.H. Levitt, Singlet and Other States with Extended Lifetimes, Encyclopedia of Magnetic Resonance (John Wiley, Chichester, UK, 2010).

[29] G. Pileio, Prog. Nucl. Magn. Reson. Spectrosc. 56, 217 (2010).

[30] S. Cavadini, J. Dittmer, S. Antonijevic, and G. Bodenhausen, J. Am. Chem. Soc. 127, 15744 (2005).

[31] A. Bornet, R. Sarkar, and G. Bodenhausen, J. Magn. Reson. 206, 154 (2010).

[32] A. Bornet, S. Jannin, J. A. Konter, P. Hautle, B. van den Brandt, and G. Bodenhausen, J. Am. Chem. Soc. 133, 15644 (2011).

[33] P. Miéville, P. Ahuja, R. Sarkar, S. Jannin, P. R. Vasos, S. Gerber-Lemaire, M. Mishkovsky, A. Comment, R. Gruetter, O. Ouari, P. Tordo, and G. Bodenhausen, Angew. Chem., Int. Ed. 49, 6182 (2010).

[34] See Supplemental Material at http://link.aps.org/ supplemental/10.1103/PhysRevLett.109.047602 for details. 